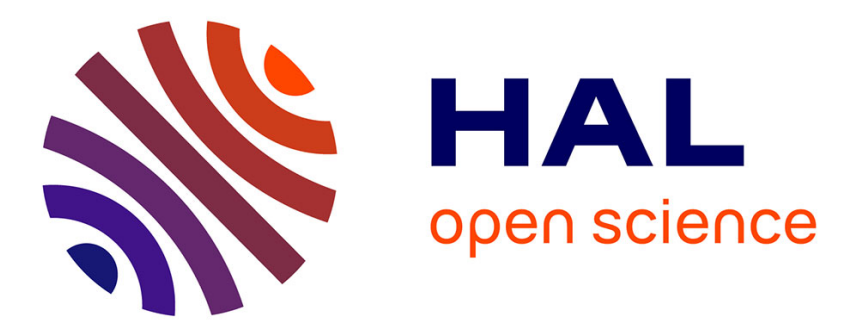

\title{
Une nouvelle géographie entre concurrence et redéploiement spatial, les migrations africaines au sein de la CEE
}

Nelly Robin

\section{- To cite this version:}

Nelly Robin. Une nouvelle géographie entre concurrence et redéploiement spatial, les migrations africaines au sein de la CEE. Revue Europeenne des Migrations Internationales, 1994, 10 (3), pp.1732. 10.3406/remi.1994.1424 . halshs-01057000

\section{HAL Id: halshs-01057000 \\ https://shs.hal.science/halshs-01057000}

Submitted on 21 Aug 2014

HAL is a multi-disciplinary open access archive for the deposit and dissemination of scientific research documents, whether they are published or not. The documents may come from teaching and research institutions in France or abroad, or from public or private research centers.
L'archive ouverte pluridisciplinaire HAL, est destinée au dépôt et à la diffusion de documents scientifiques de niveau recherche, publiés ou non, émanant des établissements d'enseignement et de recherche français ou étrangers, des laboratoires publics ou privés. 


\section{Une nouvelle géographie entre concurrence et redéploiement}

\section{spatial, les migrations africaines au sein de la CEE}

In: Revue européenne de migrations internationales. Vol. $10 \mathrm{~N}^{\circ} 3$. CERPAA CERPOD ORSTOM - Migrations

africaines. pp. 17-31.

Citer ce document / Cite this document :

Robin Nelly. Une nouvelle géographie entre concurrence et redéploiement spatial, les migrations africaines au sein de la CEE. In: Revue européenne de migrations internationales. Vol. 10 №3. CERPAA CERPOD ORSTOM - Migrations africaines. pp. 1731.

doi : 10.3406/remi.1994.1424

http://www.persee.fr/web/revues/home/prescript/article/remi_0765-0752_1994_num_10_3_1424 
Résumé

Une nouvelle géographie entre concurrences et redéploiement spatial. Les migrations africaines au sein de la CEE

Nelly ROBIN

L'espace migratoire ouest-africain constitue une réalité fluctuante qui repose sur un espace bipolaire aux fondements historiques et linguistiques et un espace engagé dans un double processus d'exclusion et de concurrences. La France, I'Italie et la Péninsule Ibérique accueillent les turbulences des migrations ouest-africaines, confrontées aux autres migrations des "Sud » au sein de la CEE et voient s'opérer un redéploiement spatial est-ouest indédit. Ces mutations remettent en cause la pertinence de la symétrie entre l'ancien espace colonial et l'espace migratoire. Comme pour bien d'autres pays du Sud, la Péninsule Ibérique s'affirme comme la « nouvelle porte d'entrée » des migrations ouestafricaines au sein de la CEE.

Abstract

A New Geography between Competitions and Spatial Redeployment. West African Migrations into EEC Nelly ROBIN

The West African migratory field constitutes a fluctuating reality which is settled on a bipolary field (with historic and linguistic foundations) and on a field engaged in a double process of exclusion and competition. France, Italy and Iberian Peninsula receive the turbulences of West African migrations confronted with other migrations from the "South » in EEC and are the witnesses of an east-west spatial redeployment un precedented. These mutations call the relevance of the symmetry between the old colonial field and the migratory field into question. As many other southern countries, the Iberian Peninsula becomes the "new gateway » for the West African migrations within the EEC.

Resumen

Una nueva geografía entre concurrencía y reorganización espacial : las migraciones de Africa del Oeste hacia la CEE

Nelly ROBIN

El espacio migratorio de los Africanos del Oeste constituye una realidad fluctuante. Se despliega en un espacio bipolar, cuyos fundamentos son historicos y linguisticos, espacio hoy encuadrado en un doble proceso de exclusión y de concurrencías. Francía, Italia y la Peninsula Iberica acogen las turbulencias de estas migraciones, confrontadas cón las otras migraciones del "Sur » en los países de la Comunidad Europea y con una reorganización espacial Este-Oeste inédita. Estas mutaciones nos obligan a reexaminar la pertinencia de la simetría entre antiguo espacio colonial y espacio migratorio. Como para otras immigraciones del «Sur », la Peninsula Iberica se afirma como la nueva puerta de entrado de los Africanos en el espacio de la CEE.

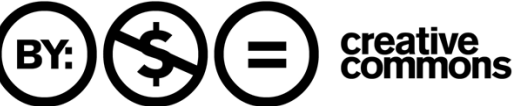


Revue Européenne des Migrations Internationales Volume $10-\mathrm{N}^{\circ} 3$ 1994

\title{
Une nouvelle géographie entre concurrences et redéploiement spatial
}

Les migrations ouest-africaines au sein de la CEE

\author{
Nelly ROBIN
}

Sous l'angle de la spatialité, l'architecture des migrations ouest-africaines (') doit êre abordée tant en terme de migrations Sud-Sud, c'est-à-dire entre les pays africains, qu'en terme de migrations Sud-Nord, c'est-à-dire entre l'Afrique de l'Ouest et l'Europe ou l'Amérique du Nord. Ces deux réalités sont étroitement imbriquées voire interdépendantes ; les migrations directes d'un pays ouestafricain vers la CEE se font rares ; le transit par un autre pays africain, qui varie suivant la période, s'impose comme une étape obligée dans un itinéraire d'émigration vers le Nord. En outre, les migrations ouest-africaines à l'intérieur de la CEE sont très actives et toujours en mutation. Le système migratoire ouest-africain ne constitue donc pas une entité autonome. Sa structure à géométrie variable dépend de la dynamique des autres systèmes migratoires " des Sud », qu'ils soient africains, asiatiques ou latino-américains, et interfere à son tour sur eux; cette relation réflexive contient toute la dynamique contemporaine des migrations ouest-africaines qu'il nous importe de décrypter.

Le prisme des migrations originaires de l'Afrique de l'Ouest au sein de la CEE, nous fait comprendre les relations de complémentarité, de dépendance, de confrontation ou d'exclusion qui existent entre les différents systèmes migratoires des "Sud". Cette mise en perspective devrait nous autoriser quelques hypothèses sur le devenir de l'espace migratoire ouest-africain. Aujourd'hui, le système migratoire ouest-africain est engagé dans une phase de transition : espace bi-polaire aux fondements historiques et linguistiques, il devient un espace multipolaire et fluctuant. Ainsi, les migrations ouest-africaines vers la CEE s'inscrivent dans un double processus de redéploiement spatial et de « concurrences ». 


\section{ARCHITECTURE DES MIGRATIONS OUEST-AFRICAINES EN CEE}

Pour saisir l'architecture des migrations ouest-africaines en CEE, nous avons recours aux statistiques d'EUROSTAT $\left(^{2}\right.$ ). Notre analyse repose sur l'analogie géographique $\left({ }^{3}\right)$ qui met les migrations ouest-africaines en perspective avec les autres dynamiques migratoires à l'échelle du continent africain ou de manière plus large à l'échelle des pays du Tiers-Monde qu'ils soient africains, asiatiques ou latino-américains et autorise une analyse de leur dynamique interne.

\section{LES MIGRATIONS OUEST-AFRICAINES ET LES AUTRES DYNAMIQUES MIGRATOIRES}

L'analyse en composantes principales des migrations internationales au sein de la CEE dessine le cadre général dans lequel doivent se mouvoir les migrations ouestafricaines et met en exergue cinq destinations essentielles; celles-ci se scindent en deux groupes qui s'opposent et se composent d'éléments hétérogènes.

GRAPHIQUE 1 : Les migrations ouest-africaines en CEE

Processus de double " concurrence », interafricaine-intercontinentale

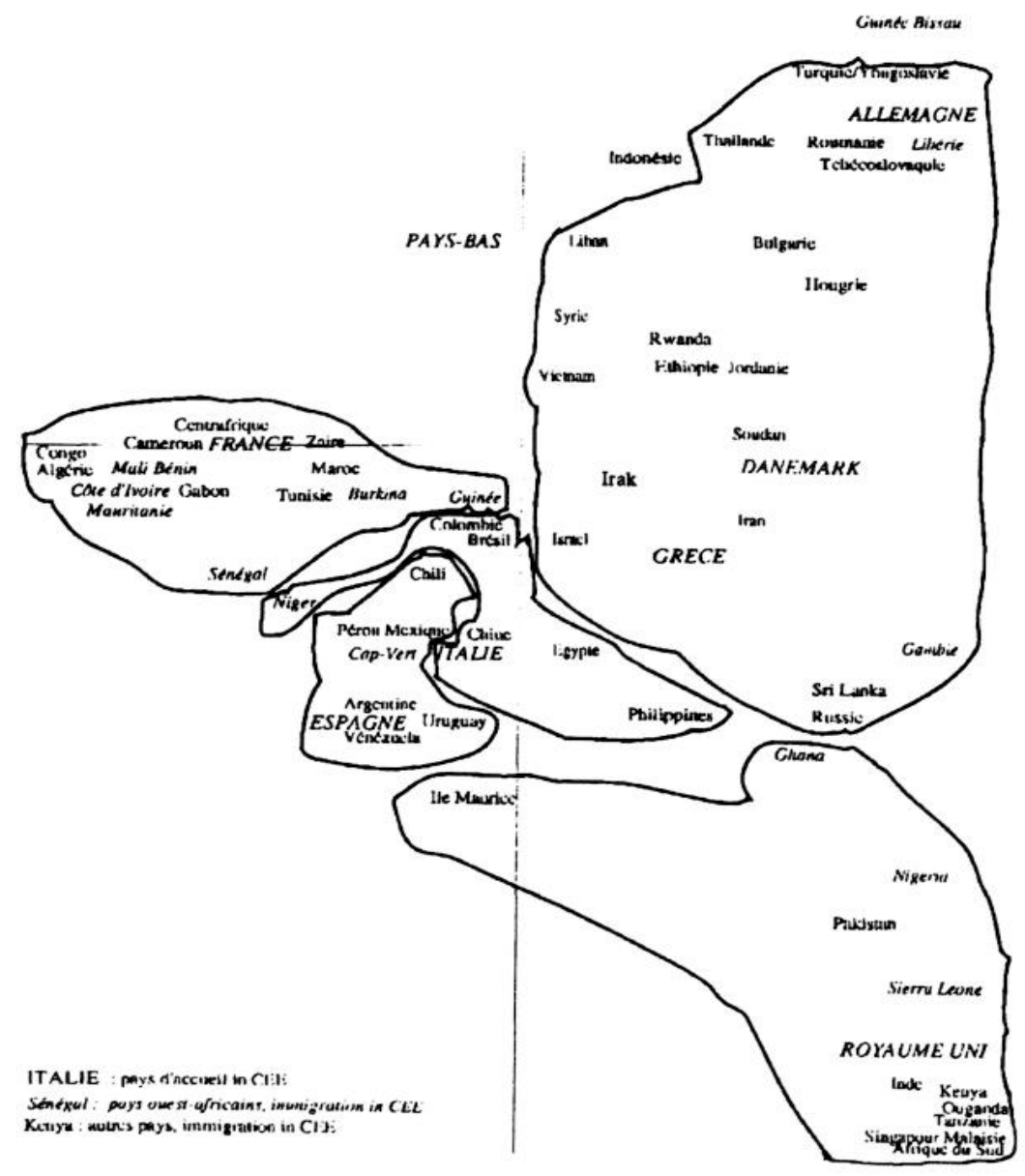


Selon les axes de l'ACP (Graphique 1), de gauche à droite, l'Allemagne et le Royaume-Uni s'opposent à la France et l'Italie, l'Espagne, le Portugal. Toutefois, l'Allemagne et le Royaume-Uni se situent aux deux extrémités opposées de l'axe vertical : ainsi, l'Allemagne accueille principalement des migrations européennes (originaires d'Europe de l'Est) ou eurasiennes (plus précisément turques) ; par contre, le Royaume-Uni reçoit surtout des migrations africaines ou asiatiques. Il s'agit de deux espaces d'émigration dichotomiques procédant de logiques très différentes, basées sur une proximité géographique ou des liens historiques et linguistiques.

A gauche de l'axe vertical se dessine une autre réalité migratoire multipolaire, scindée en trois espaces spécifiques : la France, l'Italie et la Péninsule Ibérique. La France apparaît comme le lieu d'immigration prééminent du Maghreb et de l'A frique de l'Ouest, la Péninsule Ibérique accueille l'Amérique latine, et l'Italie constitue un pôle de convergence pour l'Afrique, l'Amérique latine et l'Asie. Ce système migratoire sud-européen est le théâtre de profondes mutations réalisées ou en cours ; si hier et aujourd'hui encore, le véritable enjeu peut se situer en Italie, déjà il s'immisce en Espagne et au Portugal ; cette double translation sud-nord, de la France vers l'Italie, et est-ouest, de l'Italie vers la Péninsule Ibérique constitue un véritable défi pour les pays d'émigration du Tiers-Monde comme pour les nouveaux pays d'immigration sud-européens.

Schématiquement, l'espace migratoire de la CEE recouvre trois réalités distinctes dans le temps et dans l'espace :

- un espace d'accueil traditionnel et linguistique, en voie de stabilisation ou actif et tendant à se diversifier ; il réunit la France qui accueille essentiellement des migrations originaires d'Afrique et le Royaume-Uni qui reçoit tout d'abord des migrations d'origine africaine ou asiatique ;

- un espace singulier : l'Allemagne qui se caractérise par des migrations en provenance de l'Europe de l'Est ou du Moyen-Orient ;

- un espace instable en mutation et en construction : l'Italie et la Péninsule Ibérique particulièrement sollicitées par de nouvelles migrations africaines et asiatiques. Cet espace migratoire est conjointement animé par une immigration directe, des migrations de transit et un mouvement de redéploiement spatial sud-nord ou estouest. Les migrants d'Afrique de l'Ouest manient avec beaucoup de dextérité cette triple opportunité. Parmi les principaux pays d'immigration en Italie, hormis le Maghreb, figurent essentiellement des pays dont les premiers lieux d'immigration sont l'Allemagne ou le Royaume-Uni. Le Sénégal, dont la première destination est la France, fait ainsi exception ; il s'immisce dans un nouvel espace migratoire dont le Maroc et la Tunisie constituent des pôles de transit très actifs.

Affinons cette ébauche très générale du panorama des migrations internationales vers la CEE par une étude comparative entre les migrations ouest-africaines et les migrations originaires des autres pays africains ou de l'Asie. 


\section{LES DESTINATIONS PRIORITAIRES}

L'ACP (Graphique 1) permet de classer les pays émetteurs africains, asiatiques ou latino-américains selon leur destination prioritaire. Il s'agit d'une image synthétique de l'espace migratoire des "pays du Sud" dans lequel évolue les migrations ouest-africaines.

En France, celles-ci cohabitent avec les migrations maghrébines, bien plus nombreuses, et les migrations d'Afrique Centrale, plus rares. Les migrations tunisiennes et sénégalaises sont proches, scindées entre la France, premier pays d'immigration européen, et l'Italie. Au Royaume-Uni, la Sierra Leone présente un profil comparable non seulement à celui du Kénya, de l'Ouganda, de la Tanzanie et de l'Afrique du Sud, mais aussi de l'Inde, de Singapour et de la Malaisie. Les migrations nigérianes et ghanéennes ressemblent en ce qui concernent leur destination aux migrations pakistanaises.

De manière étonnante, en Italie, la structure migratoire du Niger est quelque peu analogue à celle de la Chine, se distinguant ainsi de celles du Sénégal, des Philippines, de l'Égypte et plus encore de la Colombie et du Brésil qui constituent les principaux pays de départ.

Parmi les pays d'Asie ou d'Afrique de l'Ouest dont les migrations vers l'Espagne sont significatives on retrouve soit des pays dont l'Italie est le premier lieu d'immigration au sein de la CEE (Philippines), soit des pays où l'Italie constitue la seconde destination en CEE (Sénégal) après les pays traditionnels d'accueil. Comme les migrations vénézuéliennes, les migrations bissau et cap-verdiennes s'orientent essentiellement vers le Portugal.

Si d'un point de vue numérique, en Espagne les migrations originaires du Sénégal et du Cap-Vert, des Philippines, de l'Inde, de la Chine, et du Pakistan, de l'Iran, du Liban et de la Syrie se situent dans une fourchette relativement étroite, au sein des autres pays de la CEE, les variations numériques sont parfois importantes. A titre d'exemple, en 1991 on comptait 3500 Burkinabé en France contre 619 900 Algériens, 2000 Sierra Léonais au Royaume-Uni contre 22000 Nigérians, 25100 Sénégalais en Italie contre 41200 Tunisiens ( ${ }^{4}$ ).

Ainsi, selon les pays d'accueil de la CEE, les migrations ouest-africaines côtoient soit presque exclusivement d'autres migrations africaines, soit simultanément des migrations d'Afrique de l'est ou du sud et des migrations asiatiques, soit de manière plus marginale des migrations latino-américaines. Elles sont par contre peu en relation avec les migrations de l'est-européen.

\section{L'ESPACE MIGRATOIRE OUEST-AFRICAIN : UNE RÉALITÉ FLUCTUANTE}

Au regard des seules migrations ouest-africaines vers la CEE (Graphique 2), trois types de pays d'accueil peuvent être isolés :

- L'Espagne, les Pays-Bas et le Danemark comptent peu de migrants ; néanmoins, pour l'Espagne, ce constat à partir des chiffres officiels est quelque peu erro- 
né et doit être révisé en fonction de l'importance inconstestée des migrations clandestines. Citons Le Monde du 24.10.1992 : «Le lieutenant-colonel Mariano de la Guardia civil étale les chiffres : 2848 immigrants illégaux arrêtés depuis 1989 dont plus de la moitié (1547) seulement en 1992 ». Parmi eux de nombreux africains qui ont transité par la Côte-d'Ivoire, le Burkina Faso, la Guinée-Bissau, le Maroc... Ainsi, Adjuma, parti du Sénégal, a vivoté pendant deux ans en Côte-d'Ivoire avant d'entreprendre la traversée du Burkina Faso, du Niger, du Sahara, de l'Algérie et du Maroc pour rejoindre l'Espagne ;

- La France, le Royaume-Uni, l'Allemagne et l'Italie constituent le principal lieu d'immigration de différents pays d'Afrique de l'Ouest avec des effectifs très variables.

- Le Portugal accueille essentiellement des migrations originaires du Cap-Vert et de la Guinée-Bissau.

GRAPHIQUE 2 : Les migrations ouest-africaines en CEE

Processus de double « concurrence », interafricaine-intercontinentale

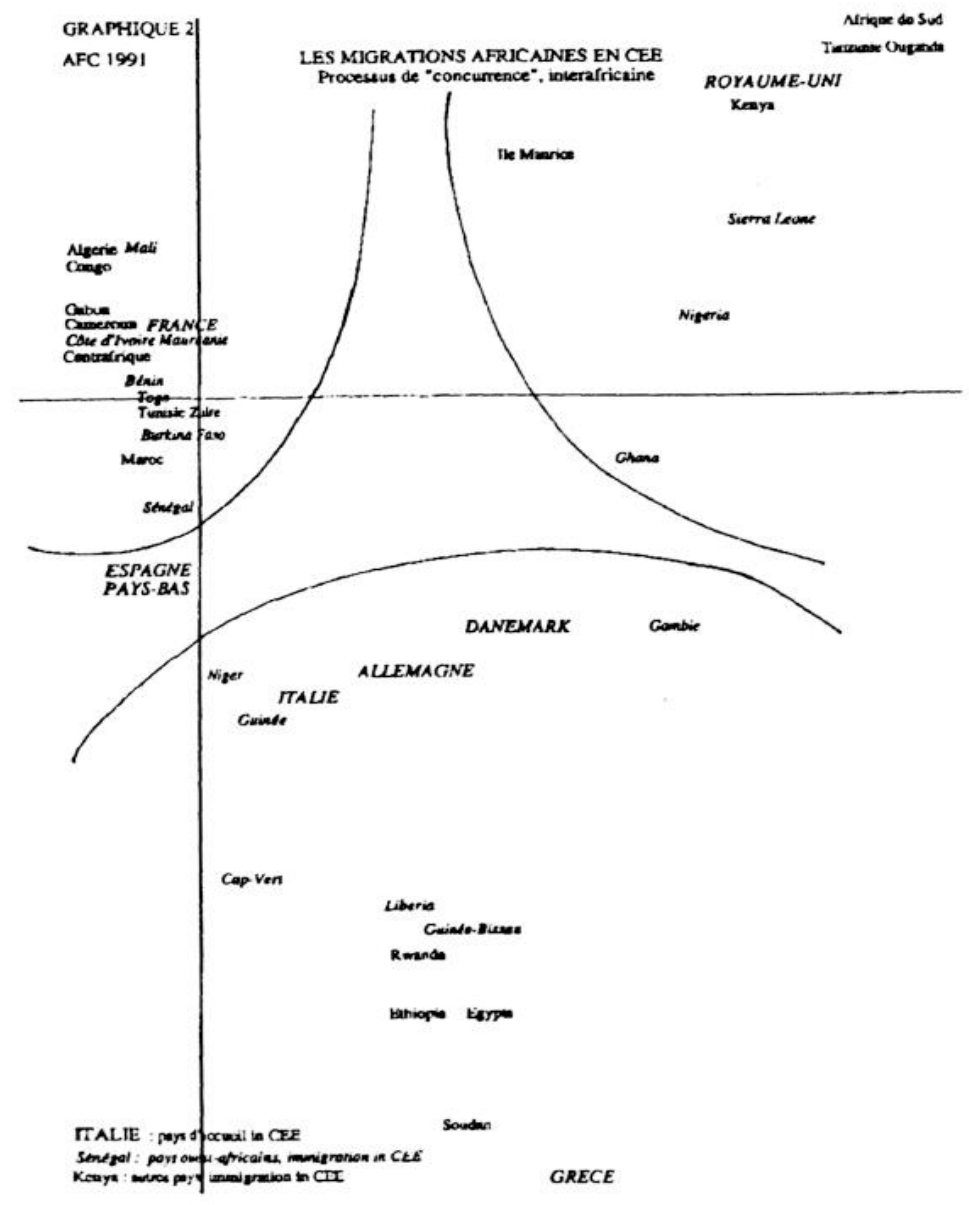




\section{Migrations africaines vers la C.E.E. dominantes des années 1990 - 1991 - 1992}
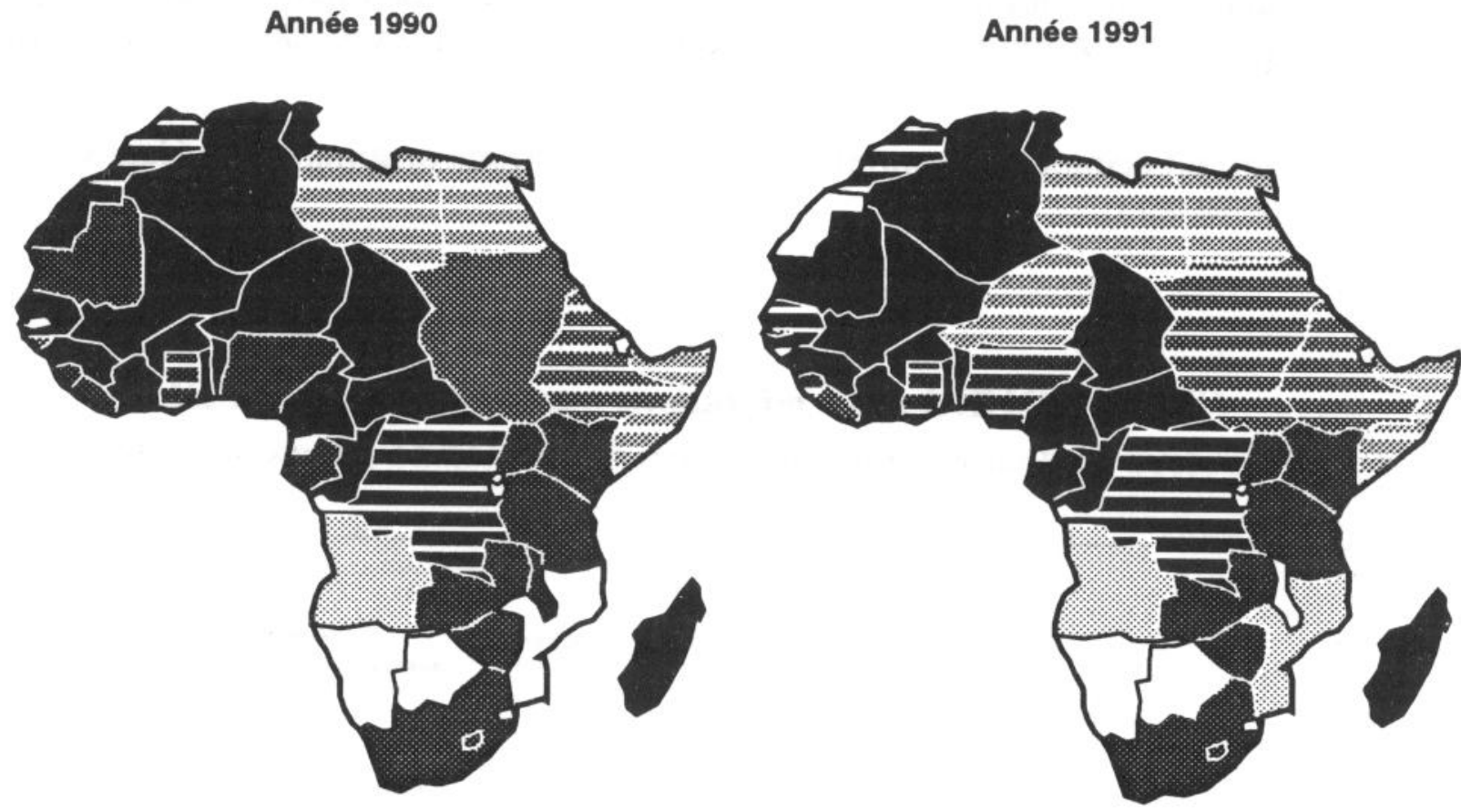

Migration vers la France

Migration vers : France-Italie ou France-Belgique

Migration vers le Royaume-Uni

Migration vers : Royaume-Uni ou Allemagne-Italie

Migration vers I'Allemagne

Migration vers : Allemagne-Italie

Migration vers I'Italie

Migration vers : Italie-Grèce ou Allemagne

Migration vers l'Espagne

Effectif non représentatif

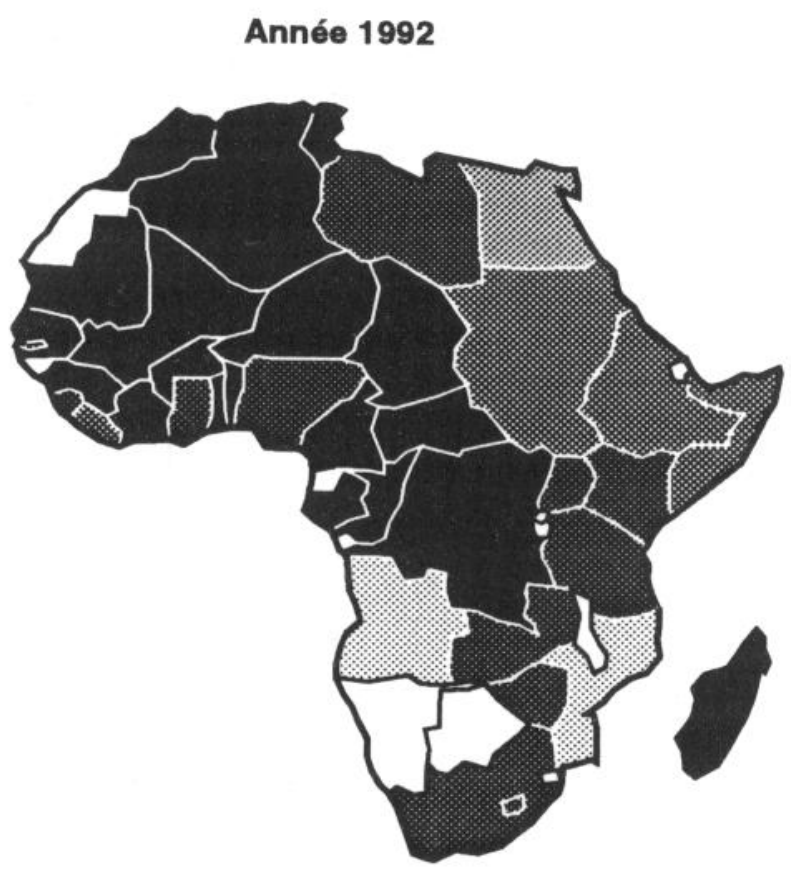




\section{L'Occupation Européenne en Afrlque au début du XXe siòcle}

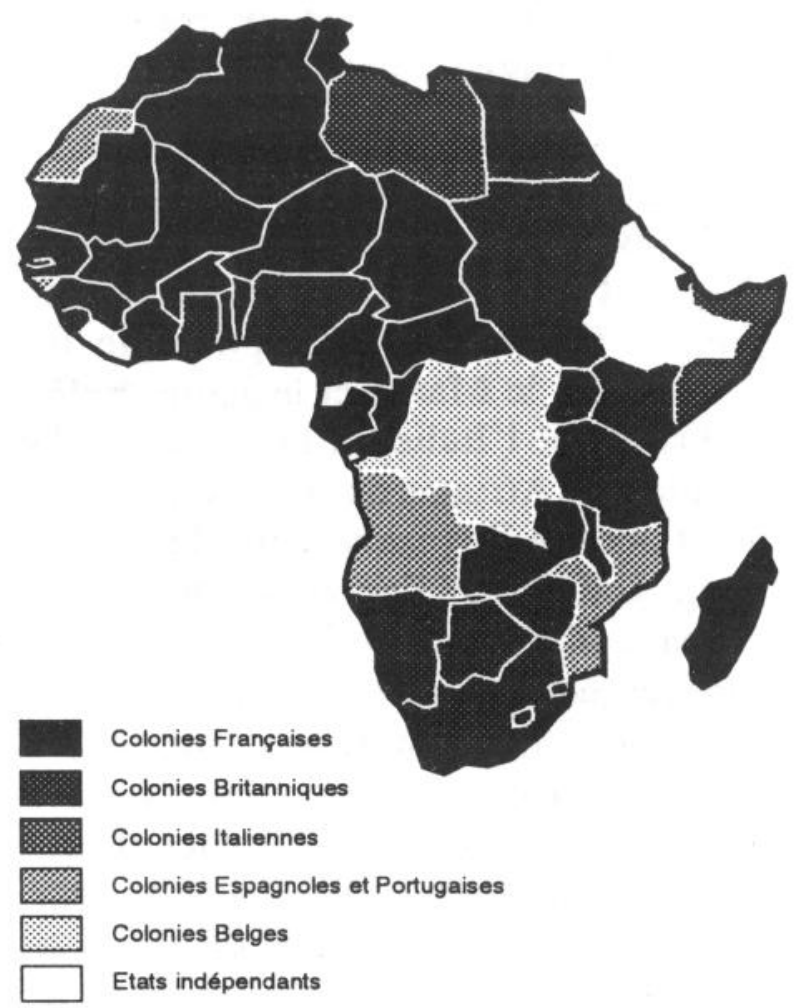

\section{Appartenance aux grandes communautés internationnales linguistiques}

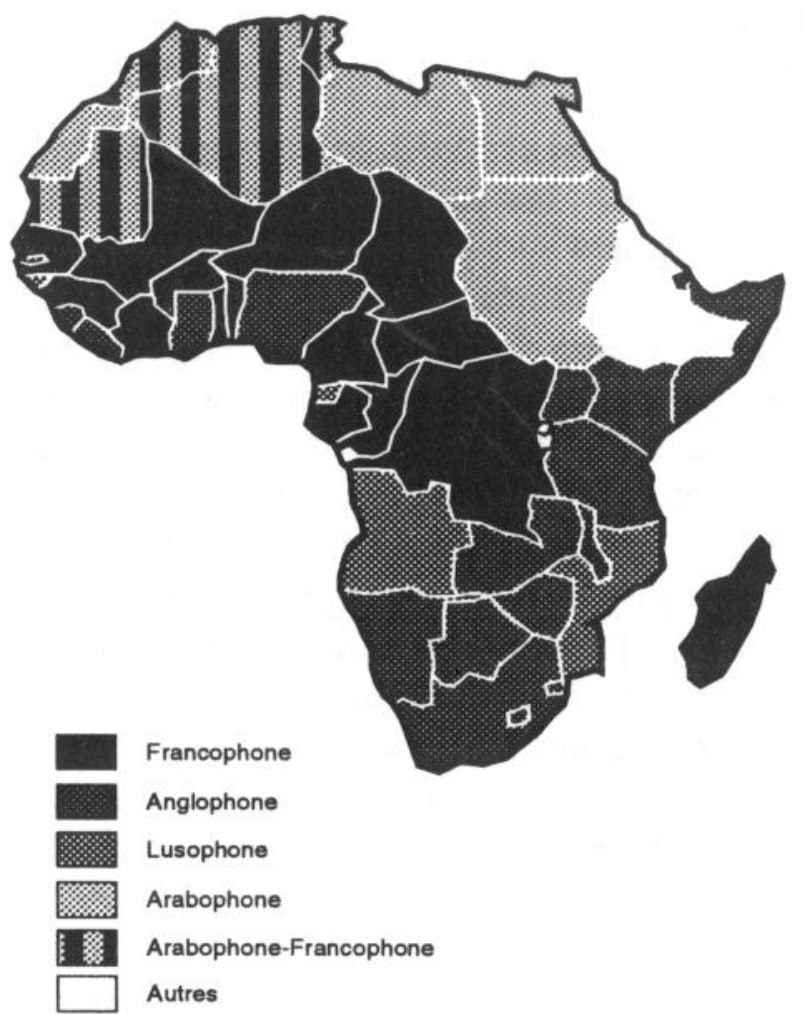

Migrations africaines vers la C.E.E. Annóe 1992

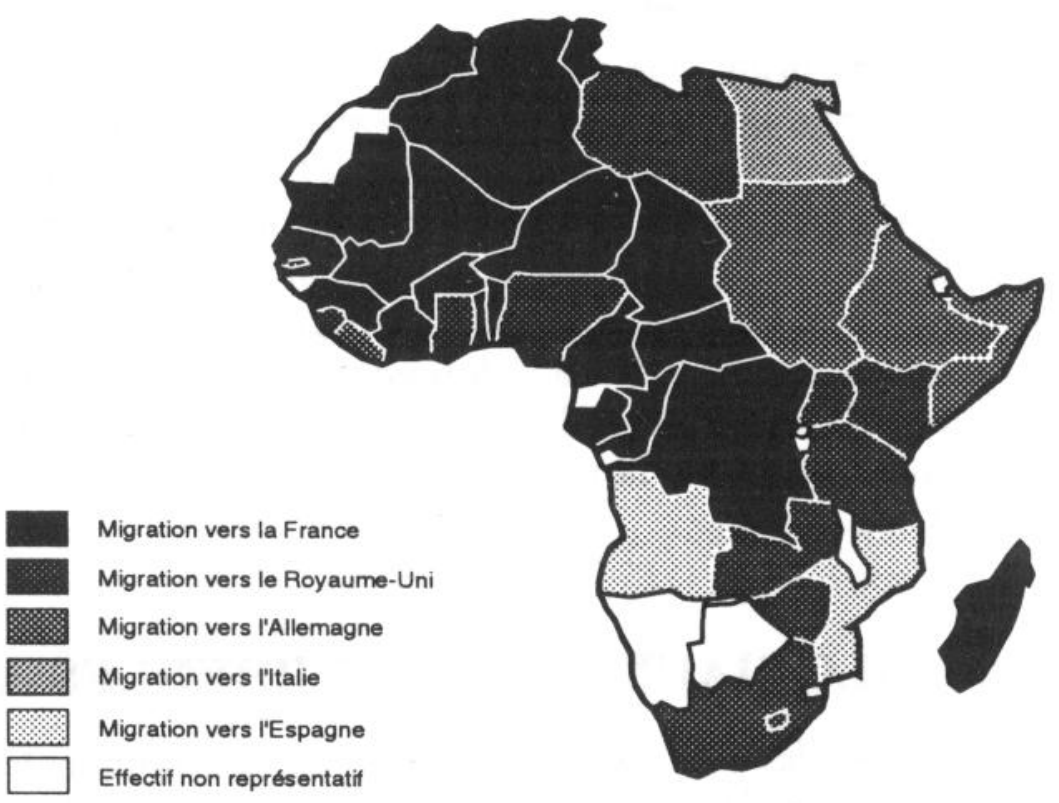


Au début des années 1990, l'espace migratoire ouest-africain se caractérise par une structure tripartite. A la périphérie, des «zones originales » qui opposent les pays qui émigrent vers le Royaume-Uni et les pays qui optent pour l'Allemagne. Au centre, une réalité sans surprise, réunit les pays qui se dirigent essentiellement voire exclusivement vers la France. En position intermédiaire, des modalités de transition correspondent aux pays qui s'orientent vers l'Italie, l'Espagne ou/et la Grèce.

Entre 1990 et 1992, le paysage des migrations ouest-africaines vers la CEE fluctue. En 1990 , les pays d'Afrique de l'Ouest privilégient essentiellement deux destinations (Carte 1) : la France et le Royaume-Uni. Le Bénin, le Burkina Faso, la Côted'Ivoire, la Guinée, le Mali, la Mauritanie, le Niger et le Sénégal s'orientent essentiellement vers la France ; l'Algérie, la Tunisie, le Cameroun, la République de Centrafrique et le Gabon rejoignent ce profil ; par contre, le Maroc et le Zaïre préferent une solution mixte entre respectivement la France et l'Italie, et la France et la Belgique. Comme l'Ouganda, le Kénya, la Tanzanie et l'Afrique du Sud, le Libéria, le Nigéria et la Sierra Leone optent principalement pour le Royaume-Uni ; le Ghana se distingue en se partageant entre le Royaume-Uni et l'Allemagne. Dès 1991 (Carte 1), quelques évolutions apparaissent : les pays d'Afrique de l'Ouest se tournent dès lors, non seulement vers la France et le Royaume-Uni mais aussi vers l'Italie et l'Allemagne. De nouveaux pays optent pour une migration mixte (Sénégal : France-Italie, Nigéria : Royaume-Uni-Allemagne), d'autres se réorientent (Niger : Italie, Libéria : Allemagne). On assiste donc à une diversification de l'espace d'immigration européen. Par contre, dès 1992 (Carte 1), l'émigration ouest-africaine vers l'Italie semble connaître quelques perturbations. On retrouve la situation de 1990, si ce n'est le Libéria qui s'oriente toujours vers l'Allemagne comme d'ailleurs le Soudan ; la Somalie et l'Éthiopie semblent préférer une situation mixte (Allemagne/Pays-Bas et Italie).

Parmi les autres pays africains quelques originalités méritent d'être signalées : l'émigration égyptienne est essentiellement orientée vers l'Italie, l'émiration libyenne s'ouvre au Royaume-Uni, et l'émigration bissau et l'émigration angolaise se dirigent prioritairement vers l'Espagne.

En terme de migrations ouest-africaines sud-nord, le Sénégal, le Ghana, le Nigéria et la Sierra Leone introduisent les dynamiques les plus complexes et les plus actives. Si le Bénin, la Côte-d'Ivoire, le Mali et le Togo suivent des itinéraires linéaires et classiques, par contre le Niger semble proposer une migration nouvelle binaire, et la Gambie ou le Libéria étonnent par leur choix. Les relations migratoires entre l'espace italien et l'espace ibérique apportent une nouvelle donne dont on ne sait encore s'il s'agit d'une migration contiguë de proximité spatiale ou une migration de substitution. En tant que pôle récepteur et de plus en plus redistributeur, l'Italie détient un rôle clef dans la reconstruction de l'espace migratoire ouest-africain au sein de la CEE.

\section{HÉRITAGES COLONIAUX ET CONCURRENCES ACTUELLES}

A travers le prisme des migrations ouest-africaines, nous entendons : faire ressortir les traits principaux des espaces traditionnels et des espaces en mutation, et comprendre les mécanismes de transfert entre ces deux espaces. 


\section{UN ESPACE BIPOLAIRE AUX FONDEMENTS HISTORIQUES ET LINGUISTIQUES}

Si l'on tente une schématisation des migrations ouest-africaines dans les pays de la CEE, différents profils apparaissent :

- un profil moyen caractérisé par une immigration relativement dispersée,

- des profils distincts, marqués par une corrélation historique ou/et linguistique

Côte-d'Ivoire, Bénin, Mali, Togo .... . . France

Nigéria, Sierra Leone .......... Royaume-Uni

Libéria ................. Allemagne

Cap-Vert, Guinée Bissau ......... Portugal

Ghana $\ldots \ldots \ldots \ldots \ldots \ldots$ Royaume-Uni et Allemagne

Deux espaces linguistiques, francophone et anglophone, quatre espaces historiques, français, britannique, allemand et portugais, qui se combinent ou s'excluent, telles sont les bases de la géographie de l'émigration ouest-africaine vers la CEE ; une superposition des cartes coloniales ou linguistiques et de la carte de l'émigration ouest-africaine est édifiante (Carte 2).

La symétrie entre l'espace africain d'émigration vers la France et l'espace colonial de l'Afrique occidentale française et de l'Afrique équatoriale française est parfaite. Deux petites nuances seulement sont à signaler qui ont néanmoins, nous le verrons, un impact sur les migrations contemporaines ; deux zones ont été sous occupation allemande vers 1885, l'une recouvre le Togo actuel et s'étend sur le Ghana, l'autre correspond au Cameroun et se prolonge sur le territoire nigérian.

Entre l'espace colonial britannique et l'espace africain d'émigration vers le Royaume-Uni la corrélation est plus nuancée. La Sierra Leone et le Nigéria comme l'Ouganda, le Kénya, la Tanzanie et l'Afrique du Sud émigrent préférentiellement vers le Royaume-Uni. La Zambie et la Namibie suivent ce schéma mais avec des effectifs très faibles. Par contre, l'Égypte et le Soudan ont rompu avec cette logique historique préférant respectivement l'Italie et l'Allemagne. En outre, l'émigration ghanéenne vers l'Allemagne est étroitement liée aux politiques allemandes parmi les plus favorables aux demandeurs d'asile en Europe.

Les anciennes colonies italiennes, la Libye et la Somalie, et les anciennes colonies portugaises, l'Angola et la Guinée-Bissau, restent fidèles à l'histoire.

États indépendants, le Libéria s'est orienté vers le Royaume-Uni et l'Éthiopie a opté pour une double migration vers l'Allemagne et l'Italie ; cette dernière peut s'expliquer par l'occupation italienne de l'Éthiopie entre 1936 et 1941.

Ainsi, l'émigration des pays de l'Afrique de l'Ouest est fortement calquée sur la géographie des anciens espaces coloniaux. Pour le reste de l'Afrique, la situation est plus nuancée, d'autres facteurs semblent interférer. 
Toutefois, l'analogie entre les pays d'émigration et les pays d'accueil s'avère plus linguistique qu'historique; s'il existe effectivement une étroite symétrie entre l'espace francophone d'émigration et l'ancien espace colonial de la France, par contre l'espace anglophone d'émigration et l'ancien espace colonial britannique connaissent quelques discordances. Ainsi, le Libéria anglophone, lié par le passé aux États-Unis, s'oriente vers l'Allemagne. La Somalie toujours fidèle à l'Italie d'un point de vue migratoire est devenue anglophone. Il faut également tenir compte des mutations linguistiques d'origine culturelle et religieuse : certains pays hier colonies britanniques (Égypte, Soudan) ou colonies italiennes (Libye) sont devenus arabophones ; en outre, l'Algérie, le Maroc et la Mauritanie appartiennent aujourd'hui à une double communauté linguistique, francophone et arabophone.

\section{UN DOUBLE PROCESSUS : ENTRE EXCLUSION ET CONCURRENCE}

Au Sud, les crises économiques (Cameroun) $\left({ }^{5}\right)$ et politiques (Nigéria, Zaïre, Congo, pour ne citer que quelques exemples des pays africains traditionnellement d'accueil) se succèdent, s'intensifient et se généralisent, et en matière de migration le protectionnisme progresse au sein des pays traditionnels d'accueil telle la Côted'Ivoire $\left(^{6}\right)$, ou des « nouveaux eldorado » comme l'Afrique du Sud $\left({ }^{7}\right)$; ceci génère au pire des expulsions $\left(^{8}\right)$ mais induit toujours une restructuration des stratégies migratoires, c'est-à-dire des espaces tissés Sud-Sud. Au Nord, simultanément, les pressions migratoires s'intensifient et les frontières se ferment sous l'effet de lois de plus en plus draconiennes. Dans ces conditions, le contact entre les migrations africaines, déjà anciennes, et les migrations venues plus récemment de l'Europe de l'Est est souvent posé en terme d'interrogation pour le devenir des migrations du sud au sein de la CEE. Pourtant, au regard de données objectives, pour les migrations ouestafricaines la question ne semble pas se situer ici, mais au niveau d'un rapport interne à la sphère des pays du sud, c'est-à-dire entre pays africains et pays asiatiques.

Ce schème migratoire repose sur la dialectique de l'exclusion et de la confrontation. Si, selon deux logiques différentes, le Royaume-Uni et l'Allemagne marginalisent les migrations ouest-africaines, par contre la France et l'Italie les situent dans un processus de concurrences spécifiques.

\section{Une réalité marginale au Royaume-Uni et en Allemagne}

Au Royaume-Uni les principales migrations observées émanent de l'entité du Commenwealth qui met en relation les pays ouest-africains anglophones (Gambie, Ghana, Nigéria, Sierra Leone), les pays de l'Afrique orientale et du Sud et certains pays asiatiques (dont les principaux sont par ordre décroissant : l'Inde, le Pakistan, le Bangladesh, le Sri Lanka, les Philippines, la Malaisie, Hong-Kong,...) et des Caraibes (la Jamaïque) ; ceci créent des concurrences au sein d'un espace dont l'assise est simultanément ou alternativement historique, linguistique ou économique. Le Royaume-Uni se caractérise par une prédominance de l'immigration asiatique $(68 \%$ des migrations venues des pays du Sud) et une représentation limitée, numériquement $(6,7 \%$ des migrations venues des pays du Sud, soit le tiers des migrations africaines) et spatialement, de l'immigration ouest-africaine ; les seules exceptions résul- 
tent d'une appartenance à l'espace anglophone. D'un point de vue des effectifs, loin derrière l'Inde ou le Pakistan, le Nigéria et le Ghana se situent dans une tranche qui réunit essentiellement des pays africains et des pays asiatiques dont les Philippines et la Malaisie. Parmi les migrations africaines au Royaume-Uni, celles du Nigéria et du Ghana sont les plus nombreuses mais leur volume reste modeste, soit environ 20000 immigrants par pays.

Leur originalité, comparés aux autres pays du Sud, peut résider dans leur structure démographique ou socioprofessionnelle ; les Africains semblent plus jeunes que les Ouest-africains ; entre ces deux entités, une symétrie existe au niveau de la structure d'âge des femmes; par contre les structures d'âge des hommes sont quelque peu discordantes. Le Nigéria et le Ghana présentent des structures d'âge très typées, composées essentiellement d'individus de 25 à 34 ans; dans les deux cas les femmes sont un peu plus jeunes que les hommes. Puisqu'il s'agit d'immigrants en âge d'activité, leur devenir au Royaume-Uni est étroitement lié à leur capacité d'investir des secteurs professionnels spécifiques et à l'évolution politico-économique des pays de l'Asie du Sud-Est.

En Allemagne, les migrations ouest-africaines restent marginales. Elles se trouvent en concurrence avec l'Asie du Sud-est voire les pays du Moyen-Orient, alors que l'immigration des pays de la CEE reste prédominante.

\section{La France, l'Italie et la Péninsule Ibérique : les turbulences des migrations ouest-africaines au sein de la CEE}

\section{a) Un espace migratoire traditionnel en devenir}

Au sein de la CEE, la France demeure le pays de l'immigration africaine (1 652900 ) et à fortiori ouest-africaine $(128198$, soit environ $8 \%$ des migrations africaines), et c'est sans nul doute en France que les migrations ouest-africaines sont le plus en contact avec l'ensemble des migrations africaines. Numériquement, elles se situent entre les migrations originaires du Maghreb et les migrations de l'Afrique Centrale. Toutefois, leur volume est très différent d'un pays à l'autre ; le Sénégal et le Mali, premiers pays d'immigration ouest-africaine, sont dans une tranche où ils côtoient essentiellement des pays est-européens (Yougoslavie, Pologne) et surtout asiatiques (Cambodge, Laos, Vietnam); les pays d'Asie du Sud-Est réunissent à eux seuls un volume de migrations supérieur à celui de l'ensemble des dix-sept pays de l'Afrique de l'Ouest. Les autres migrations ouest-africaines sont plus modestes ; pour ne prendre que quelques exemples, l'immigration ivoirienne (17 000) s'intercale entre le Cameroun et l'Iran, l'immigration mauritanienne (6 600) entre le Chili et le Brésil, l'immigration togolaise (5 700) entre la Roumanie et l'Égypte, l'immigration béninoise ( 3900 ) entre la Colombie et le Gabon, les immigrations ghanéens et burkinabés (2 500-2 700) entre Israël et le Mexique et l'immigration nigérienne ( 1400 ) entre les Philippines et le Vénézuela. Dans ce contexte, les migrations ouestafricaines sont soumises à des pressions multiples d'ordre géographique ou politique ; la France est traditionnellement un espace d'accueil pour de nombreux pays du Sud, ce qui laisse d'autant moins de latitude à l'immigration ouest-africaine. En outre, les politiques françaises en matière de migration de plus en plus protection- 
nistes et les crises politico-économiques qui secouent les pays du Sud et plus particulièrement ces derniers temps les pays africains accentuent les concurrences potentielles ; ainsi, certains pays d'accueil pour les migrations ouest-africaines sud-sud deviennent des concurrents tant en terme de migrations sud-nord que de migrations sud-sud. Face à ces nouvelles réalités restrictives, il est impératif pour l'espace migratoire ouest-africain de devenir pluriel et mobile.

\section{b) Un redéploiement spatial est-ouest inédit}

Le glissement opéré ou en cours de l'immigration ouest-africaine des pays traditionnels d'accueil vers l'Italie, l'Espagne et le Portugal va exposer certains pays africains à un processus de concurrences. Ce redéploiement spatial nord-sud au sein de la CEE est le témoin d'une pression migratoire très forte émanant conjointement de l'Amérique latine, de l'Asie et de l'Afrique. Le Sénégal est le premier pays d'immigration ouest-africaine en Italie, il est suivi de très loin par le Ghana et le Nigéria, puis par la Mauritanie et le Cap-Vert, et enfin la Côte-d'Ivoire et le Niger. Numériquement, le Sénégal est ainsi très proche des Philippines, eux-mêmes largement précédés par le Maroc et la Tunisie. Toutefois, des similitudes intéressantes apparaissent entre l'immigration tunisienne et l'immigration sénégalaise en Italie, bien que décalées dans le temps. Ces deux pays se caractérisent par une migration traditionnelle vers la France à laquelle succède depuis quelques années une migration vers l'Italie ; cette mutation s'est opérée sous l'influence d'une proximité géographique directe pour la Tunisie. Les migrants du Sénégal quant à eux viennent en Italie après un transit en France, dans un autre pays africain (Côte-d'Ivoire, Burkina Faso, Guinée voire Cameroun) selon les opportunités législatives ou dans un pays maghrébin (Maroc, Tunisie). En outre, le Sénégal et la Tunisie passent progressivement d'une migration de clandestins à une migration de regroupement familial.

A ce glissement sud-nord de l'immigration ouest-africaine au sein de la CEE succède, sous l'effet de mesures protectionnistes en Italie $\left({ }^{9}\right)$, une translation est-ouest sur la façade méditerranéenne. En Espagne, le Sénégal et les Philippines apparaissent comme les précurseurs d'un nouvel espace migratoire sud-européen; les itinéraires qui les conduisent jusqu'à la Péninsule Ibérique semblent très diversifiés et encore très fluctuants (migration directe, transit par l'Italie ou le Maroc,...). Ces nouvelles migrations venues "des suds » vers la Péninsule Ibérique animent des relations migratoires entre l'Italie et l'Espagne ; ces migrations s'inscrivent-elles dans un processus de contiguité spatiale, d'interactivité ou de simple substitution ? Le phénomène est encore jeune et instable, les éléments de réponse restent donc un peu diffus. Quelques exemples précis nous éclairent néanmoins sur les dynamiques migratoires développées par les populations ouest-africaines qui souhaitent rejoindre la Péninsule Ibérique ; celles-ci peuvent ainsi transiter soient simplement par la France ou le Maroc, soit successivement par la Guinée Bissau ou les Canaries puis le Portugal, soit indifféremment par la Côte-d'Ivoire, le Burkina Faso, le Cameroun, la Libye, la Tunisie avant de rejoindre l'Italie via l'Espagne. A l'extrémité est de la façade méditerranéenne de la CEE, la Grèce joue un rôle semblable à celui de l'Espagne et du Portugal pour d'autres zones d'émigration. 
Ces mutations essentielles de l'espace migratoire ouest-africain au sein de la CEE remettent en cause la pertinence de la symétrie entre l'ancien espace colonial et l'espace migratoire. Comme pour bien d'autres pays du Sud $\left({ }^{10}\right)$, la Péninsule Ibérique s'affirme comme la « nouvelle porte d'entrée » des migrations ouest-africaines au sein de la CEE. Cette restructuration de l'espace migratoire ouest-africain au Nord implique de nouvelles stratégies en amont ; en Afrique, d'autres pays de transit émergent dont la durée de vie est de plus en plus éphémère. Pour répondre au contexte politique et économique de plus en plus fragile, les migrants sont contraints d'élaborer des stratégies migratoires offrant de multiples opportunités, alternativement mises en veille ou réactivées selon la conjoncture. Toutefois, il est indéniable qu'au Sud comme au Nord, les espaces potentiels d'immigration se raréfient. Pourtant, l'aspiration des populations à migrer ne cesse de croître. Dans cette perspective, une relance de la croissance économique des pays de l'Afrique de l'Ouest à moyen terme s'avère de plus en plus impérative. Au Ghana, le patronat et le gouvernement qui s'organisent pour "se lancer sur la voie tracée par les dragons d'Asie du Sud-Est (") semblent l'avoir compris. Une stabilisation politique et un renouveau économique des pays africains paraissent d'autant plus urgents que l'essentiel des migrations ouest-africaines ne s'oriente pas vers les pays du Nord mais vers d'autres pays d'Afrique tels que le Gabon, le Zaïre ou le Congo particulièrement éprouvés au cours des derniers mois. 


\section{Notes et références bibliographiques}

(1) Afrique de l'Ouest, sur la base des 16 pays de la Communauté Économique de l'Afrique de l'Ouest : Bénin, Burkina Faso, Cap-Vert, Côte-d'Ivoire, Gambie, Ghana, Guinée, Guinée-Bissau, Libéria, Mali, Mauritanie, Niger, Nigéria, Sénégal, Sierra Leone et Togo.

(2) EUROSTAT : Office Statistique des Communautés Européennes.

Population by nationality 1990 (12.12.1992), Population by nationality on 1 january 1991 (05.02.1993), Population by citizenship on 1 january 1992 (25.05.1994).

Nous avons confronté ces données à d'autres sources, puis apporté les corrections éventuellement nécessaires pour disposer d'une base de données aussi fiable que possible.

(3) Pour apprécier la dynamique des relations dans laquelle s'inscrit les migrations ouest-africaines parmi l'ensemble des migrations au sein de la CEE, nous avons opté pour l'analyse en composante principale (cf. graphique 1). La lecture du rapport entre les différents espaces migratoires reposent alors sur l'analogie migratoire ; les lieux ont les propriétés des objets qui s'y trouvent et les distances sont facteurs de ressemblance ou de dissemblance ; les concentrations de points définissent des zones opposables les unes aux autres.

(4) Margherita Boniver, ministre italien chargé de l'immigration en 1992, estimait qu'il y avait 40000 Sénégalais en Italie sur 1400000 étrangers (légaux et clandestins); les chiffres officieux dépassent les 40000 Sénégalais en Italie.

(5) Face à la crise économique qui sévit au Cameroun, des ressortissants sénégalais ont demandé au gouvernement du Sénégal à être rapatriés ; ils sont arrivés à Dakar le 22.09.1994.

(6) En mai 1990, la Côte-d'Ivoire instaure un visa pour l'entrée des étrangers : « Pour entrer en Côted'Ivoire, tout étranger doit être muni : 1. Des documents et visas exigés par les conventions internationales et les réglements en vigueur, ..., 3. Des documents nécessaires à l'exercice d'une activité professionnelle s'il se propose d'en exercer une. La production des documents, visas, et justifications prévus aux alinéas ci-dessus confere le droit d'entrer sur le territoire ivoirien », in Journal Officiel du 29 mai 1990 ; loi n $90-437$, Côte-d'Ivoire.

(7) «L'ambassade du Sénégal à Prétoria vient de rendre compte aux autorités sénégalaises de la récente mesure prise par le ministre sud-africain de l'Intérieur, M. Mangosutu Buthelezi, de procéder à l'arrestation et à l'expulsion de tous les étrangers qui résident illégalement dans ce pays.... Cette présence est perçue par les autorités sud-africaines comme une menace pour la mise en cuvre du programme de reconstruction et de développement de leur pays », in Le Soleil, le 27-28.08.1994, Sénégal.

(8) Sénégalais expulsés de Zambie en février 1992, Expulsions de Ghanéens de Côte-d'Ivoire en novembre 1993, Expulsions des Sénégalais et des Guinéens de Gambie en novembre 1993, pour ne citer que quelques exemples.

(9) Loi Martelli, avril 1990.

(10) «Selon les chiffres de l'Institut National de la Statistique et de l'Informatique (INEI), le nombre de Péruviens qui se sont rendus en Espagne en 1992 est de 6871 personnes. En comparant ce chiffre à celui des années précédentes, on constate qu'il est en hausse », in Revue Perù n 18, janvier 1994, Lima.

(11) In « Ghana, une diversification qui porte ses fruits ", Jeune Afrique Economie, $n^{\circ} 183$, septembre 1994 , p. 66. 
Une nouvelle géographie entre concurrences et redéploiement spatial. Les migrations africaines au sein de la CEE

\section{Nelly ROBIN}

L'espace migratoire ouest-africain constitue une réalité fluctuante qui repose sur un espace bipolaire aux fondements historiques et linguistiques et un espace engagé dans un double processus d'exclusion et de concurrences. La France, l'Italie et la Péninsule lbérique accueillent les turbulences des migrations ouest-africaines, confrontées aux autres migrations des « Sud * au sein de la CEE et voient s'opérer un redéploiement spatial est-ouest indédit. Ces mutations remettent en cause la pertinence de la symétrie entre l'ancien espace colonial et l'espace migratoire. Comme pour bien d'autres pays du Sud, la Péninsule lbérique s'affirme comme la "nouvelle porte d'entrée " des migrations ouest-africaines au sein de la CEE.

\section{A New Geography between Competitions and Spatial Redeployment. West African Migrations into EEC}

\section{Nelly ROBIN}

The west africain migratory field constitute a fluctuating reality wich is settled on a bipolary field (with historic and linguistic fundations) and on a field engaged in a double process of exclusion and competition. France, Italy and Iberian Peninsula receive the turbulences of west african migrations confronted with other migrations from the "South in EEC and are the witnesses of an east-west spatial redeployment un precedented. These mutations call the relevance of the summetry between the old colonial field and the migratory field into question. As many other southern countries, the Iberian Peninsula becomes the "new gateway " for the west african migrations within the EEC.

Una nueva geografía entre concurrencía y reorganización espacial : las migraciones de Africa del Oeste hacia la CEE

\section{Nelly ROBIN}

El espacio migratorio de los Africanos del Oeste constituye una realidad fluctuante. Se despliega en un espacio bipolar, cuyos fundamentos son historicos y linguisticos, espacio hoy encuadrado en un doble proceso de exclusión y de concurrencias. Francia, Italia y la Peninsula Iberica acogen las turbulencias de estas migraciones, confrontadas cón las otras migraciones del "Sur „ en los paises de la Comunidad Europea y con una reorganización espacial Este-Oeste inédita. Estas mutaciones nos obligan a reexaminar la pertinencia de la simetría entre antiguo espacio colonial y espacio migratorio. Como para otras immigraciones del "Sur „, la Peninsula Iberica se afirma como la nueva puerta de entrado de los Africanos en el espacio de la CEE. 